\title{
INCLUSIÓN Y DESARROLLO SOCIAL EN LA AGENDA $Y$ LOS PLANES DE CIENCIA, TECNOLOGÍA E INNOVACIÓN DE BRASIL Y URUGUAY (2003-2015)*
}

\author{
Gabriela Cirino $^{* *} /$ Amílcar Davyt ${ }^{* * *}$
}

\section{RESUMEN}

Se pretende contribuir a la reflexión sobre cambios y continuidades de las políticas de ciencia, tecnología e innovación (Сті) en América Latina, buscando entender los efectos de la introducción, en las prácticas políticas recientes, de modelos que vinculan la CTI con los problemas de inclusión y desarrollo social. Para ello, se analizan comparativamente las experiencias de generación de planes estratégicos de CTI de Brasil y Uruguay en el período 2003-2015. Luego de presentar las principales concepciones teóricas sobre políticas CTI, se analizan las formas en que se aborda el tema de la inclusión y el desarrollo social en los estudios, propuestas, planes y estrategias nacionales de CTI en cada país. Se analizan, después, los instrumentos de política y presupuestos implementados en el período. Finalmente, se comparan ambos procesos, destacando las similitudes y diferencias detectadas. Se concluye que el contexto político favorable para la incorporación del tema de Cті para la inclusión y el desarrollo social, propiciado por la asunción de gobiernos progresistas y la incorporación en sus agen-

* Este artículo es parte del trabajo de la tesis "Inclusión y desarrollo social en las políticas de ciencia, tecnología e innovación de Brasil y Uruguay. Un estudio comparado”, con la que se obtuvo el título de Magíster en Estudios Contemporáneos de América Latina de la Facultad de Ciencias Sociales, Universidad de la República. La tesis se realizó con el apoyo de una beca de maestría de la Agencia Nacional de Investigación e Innovación (ANII).

** Departamento de Trabajo Social de la Universidad de la República (Uruguay). Correo electrónico: <cirinoga@gmail.com>.

*** Unidad de Ciencia y Desarrollo de la Facultad de Ciencias, Universidad de la República (Uruguay). Correo electrónico: <amilcardavyt@gmail.com>. 
das de dichos temas como fundamentales, no fue suficiente para consolidar una política efectiva y duradera en la materia, más allá de su incorporación en el discurso, en documentos oficiales y en planes de ambos gobiernos.

\author{
PALABRAS CLAVE: POLÍTICAS CTI - INCLUSIÓN Y DESARROLLO SOCIAL - \\ PLANES ESTRATÉGICOS - BRASIL Y URUGUAY
}

\title{
INTRODUCCIÓN
}

Es bastante aceptada entre especialistas del campo de estudios sociales y políticos de la ciencia y la tecnología, la idea de que los modelos de políticas de ciencia, tecnología e innovación (РСTI) prevalecientes en América Latina en las últimas décadas se han ido modificando desde una concepción orientada a la competitividad y la creación de capacidades básicas en ciencia y tecnología, hacia la consideración del papel que estos elementos pueden jugar en relación a los problemas de inclusión y desarrollo social (Velho, 2011; Casas et al., 2014).

La relación entre cambio tecnológico e inclusión social se ha convertido en un tema de debate académico y objeto de políticas públicas de desarrollo en la última década (Thomas et al., 2015: 2). Diversos organismos internacionales -BID, BM, PNUD- se han preocupado por la temática y comenzado a promover líneas de acción en relación al abordaje de problemas sociales a través de políticas de ciencia y tecnología. Muchos gobiernos de la región han comenzado a incluir estrategias y programas en esta dirección.

Una característica de la mayoría de los países latinoamericanos es la consolidación de marcos jurídicos para CTI; esto se da en varios de ellos en la década de 1970 pero presentan un nuevo impulso luego del 2000, en vinculación a la asunción de gobiernos progresistas ${ }^{[1]} \mathrm{y}$ a la importancia que

[1] Concepto por medio del cual históricamente se designó desde la izquierda marxista a los programas y las prácticas constitutivas de los gobiernos y las fuerzas sociales y políticas socialdemócratas, populistas nacional-populares que buscaban reformar al capitalismo introduciendo dosis de intervención y regulación estatal y de redistribución de la riqueza (Gaudichaud et al., 2019: 9). Lanzaro (2008) define a algunos de estos gobiernos latinoamericanos de inicios del siglo xxi como socialdemocracia criolla, aludiendo a que se emparentan con fórmulas políticas de las experiencias socialdemocráticas tardías europeas. Presentan un fuerte grado de institucionalización y vínculos estrechos con el movimiento sindical, sufrieron procesos de moderación de sus posturas e ideología hacia el centro del 
estos le atribuyen a la CTI como elemento dinamizador de las políticas económicas y sociales (Casas et al., 2014).

Se pretende aquí aportar al debate sobre cambios y continuidades de las políticas de ciencia, tecnología e innovación en la región, para entender la introducción en la práctica política de modelos que vinculan la CTI con los problemas de inclusión y desarrollo social. Para arribar a este objetivo, se realiza un estudio comparado de dos países de la región, Brasil y Uruguay, analizando las experiencias de generación y consolidación de estrategias y planes de CTI en ambos países en el período 2003-2015, así como los instrumentos de política y los presupuestos derivados. Se busca aportar a la comprensión de estos procesos mediante el análisis de sus semejanzas y diferencias, con el fin de obtener aprendizajes para formular futuras políticas.

A pesar de ser bien diferentes en términos generales, los países seleccionados comparten la característica de haber introducido los temas de inclusión y desarrollo social en sus agendas de PCTI a principios de este siglo, con la asunción de gobiernos progresistas.

En primer lugar, se presentan brevemente los aspectos conceptuales de los cuales se parte para el análisis: las principales concepciones teóricas sobre políticas de ciencia, tecnología e innovación, diferenciando entre los modelos clásicos, sistémicos y los matices que adquieren estos modelos en el contexto latinoamericano.

Luego se analiza, por un lado, la creación y consolidación de la denominada Política de Ciencia y Tecnología para el Desarrollo Social (рстDs) en Brasil, con la creación de la Secretaría Nacional de Ciencia y Tecnología para la Inclusión Social (sECIS) y los documentos, planes y leyes que impulsaron su creación. Por otro lado, se analizan los principales documentos que guían el proceso de consolidación del Plan Estratégico Nacional en CTI (PENCTI) para Uruguay. Finalmente, se establece una comparación entre ambos procesos, destacando la forma en la cual es introducido y considerado el tema de la inclusión y desarrollo social.

Las consideraciones finales intentan, en última instancia, contribuir al perfeccionamiento de la futura elaboración e implementación de políticas, programas e instrumentos de CTI para la inclusión y desarrollo social en nuestros países.

espectro político y pasaron a actuar en sistemas democráticos efectivos y competitivos, aceptando las restricciones políticas y económicas impuestas por el sistema capitalista imperante (Lanzaro, 2008). 


\section{POLÍTICAS DE CIENCIA, TECNOLOGÍA E INNOVACIÓN Y SU VINCULACIÓN CON EL DESARROLLO Y LA INCLUSIÓN SOCIAL}

El desarrollo científico-tecnológico, como fenómeno que incide en los procesos de inclusión / exclusión social, puede jugar un rol fundamental en el análisis de una estrategia para la resolución de los problemas sociales que afectan hoy a los países latinoamericanos (pobreza, desigualdad social, desempleo, etc.). La producción de alimentos, vivienda, energía, transporte, para la inclusión de grandes grupos humanos que hoy se encuentran excluidos implica una preocupación por la orientación que se le puede imprimir al desarrollo de políticas de cтi en la región (Albornoz et al., 2015: 6).

Para analizar estas políticas, realizamos una distinción entre los modelos conceptuales lineales de mediados de siglo pasado, los sistémicos, desarrollados a partir de la pasada década de 1980 y lo que identificamos como abordajes conceptuales recientes en сті desde América Latina. Enfatizamos en las características e implicancias para el desarrollo de políticas de сті de estas concepciones.

El modelo lineal se desarrolla a partir de la Segunda Guerra Mundial, cuando se empieza a dar gran importancia a las relaciones entre el avance de la ciencia y la tecnología y sus posibles beneficios y contribuciones al cambio económico y social. Esto se tradujo en el impulso de las primeras "políticas científicas", tanto en países industrializados como en periféricos (Albornoz, 2007).

La PCTI surgida en la posguerra se desenvuelve basada en un concepto de "modelo lineal": de la investigación básica a la aplicada, al desarrollo experimental y a la innovación tecnológica; por último, al crecimiento y desarrollo económico y social. Se comienzan a ejecutar políticas con el foco puesto en el desarrollo de la investigación y la formación de recursos humanos, lo que se llamó política centrada en la oferta o política ofertista (Herrera, 1973).

Este modelo lineal se basa en el concepto de autonomía de la ciencia con respecto a los procesos sociales; se extrapola a los países en desarrollo una imagen neutral y lineal del desarrollo científico y el modelo normativo de ella derivado.

En la década de 1970 comienza a abandonarse la visión del investigador con total autonomía y se empieza a considerar la necesidad de dirigir la ciencia hacia intereses de desarrollo nacional. Se sigue concibiendo de forma lineal la relación entre ciencia y tecnología, pero se pone énfasis en la demanda y no en la oferta de conocimiento. Se deja de entender a la ciencia básica como impulsora de la tecnología y pasa a ser el mercado y las 
necesidades de los usuarios los que impulsan el desarrollo científico (Velho, 2011:7). Además, se entiende necesario un papel más activo de la sociedad en la determinación de la orientación de la ciencia y la tecnología.

A partir de la década de 1980, la noción de que la innovación y el progreso técnico son resultado de un conjunto complejo de relaciones entre actores que producen, distribuyen y aplican varios tipos de conocimiento, en una manera sistémica, es fuertemente promovida por la Organización para la Cooperación y el Desarrollo Económicos (OCDE, I 997). Esta concepción se vuelve el marco predominante de las políticas CTI desde finales de dicha década y se funda en trabajos empíricos que mostraban la naturaleza contingente y socialmente localizada de los hechos científicos y entendían a la ciencia como construcción social (Velho, 2011).

Se postula una innovación que tome en cuenta el sistema diverso de actores en el que se produce. Se evita su aislamiento de las otras actividades sociales y económicas, ya que se entiende como un proceso social e interactivo en un entorno social sistémico (Jonhson y Lundvall, 1994). En él, diferentes organizaciones -instituciones de CTI y de educación, empresas, usuarios, el Estado-determinan conjuntamente la intensidad y la dirección del cambio tecnológico (Lundvall, 1992).

En el marco de estos enfoques se desarrollaron diversos modelos explicativos de la relación CTI-sociedad. Todos ellos enfatizan en la necesidad de interacción entre los diversos actores del proceso de producción del conocimiento científico, compartiendo la idea de que la trama social influye en la concepción y producción del conocimiento, y no solo en su utilización. Además, explicitan de diferentes maneras que la concepción del conocimiento científico y tecnológico como determinante para el crecimiento económico lleva, por un lado, a generar cambios en la organización interna de la ciencia y, por otro, a la transformación de las relaciones entre la ciencia y otras esferas como la económica y política, cambiando a su vez las relaciones entre ciencia, tecnología y sociedad (Arancibia, 2017: 17-18).

A principios de este siglo surge la preocupación académica sobre las posibilidades del vínculo entre CTI y desarrollo e inclusión social en América Latina, producto de la constatación de las persistentes dificultades para generar procesos de desarrollo, los problemas de desigualdad en la región (CEPAL, 2014) y el descreimiento, de una parte de la comunidad académica, en la hipótesis que relaciona de manera lineal ciencia, tecnología e innovación con crecimiento económico y su consecuente derrame hacia el desarrollo social.

De esta forma, diversas corrientes coinciden en impugnar (con matices) la posibilidad de que el vínculo entre desarrollo económico e inclusión social se produzca de forma automática en el contexto latinoamericano. 
Estas corrientes concuerdan en rechazar la hipótesis de que el papel fundamental y exclusivo de la cTI en el desarrollo es colaborar a revertir la brecha estructural de productividad entre los países y de la existencia de un automatismo en el vínculo entre ciencia, tecnología e innovación, crecimiento económico y reversión de la exclusión social (Alzugaray et al., 2013: 32).

En este marco, gana peso la afirmación que sostiene que la CTI puede y debe contribuir a revertir la exclusión social, así como reducir la pobreza y la desigualdad en las sociedades latinoamericanas. Así, se despliega un conjunto de propuestas teóricas que dan lugar a distintos proyectos y redes de investigación nacionales e internacionales. Es posible identificar, en principio, tres enfoques conceptuales influyentes en el contexto latinoamericano contemporáneo sobre estos aspectos.

La concepción de "innovación inclusiva" desarrollada por algunos organismos internacionales (OECD, BM, UNCTAD), que colocan en sus discursos la exclusión social, se centra en la necesidad de inclusión en el mercado de las personas pobres por la vía de la producción y el acceso al consumo (вм, 2013).

La propuesta de "sistemas de innovación socialmente orientados", de Judith Sutz y colaboradores de la Universidad de la República (Uruguay), plantea que el principal motor de la exclusión social son las asimetrías basadas en conocimiento y propone para su superación una noción sistémica en la que participarían diversos actores de forma articulada, procurando vincular necesidades y oportunidades técnicas para contribuir a la reducción de las formas de la desigualdad (Sutz, 2010).

Por último, un tercer enfoque conceptual es el de tecnología social, que cuenta con dos orientaciones. Una de ellas es desarrollada por Renato Dagnino y sus colaboradores de la Universidade Estadual de Campinas (Brasil), y la otra por Hernán Thomas y su equipo de la Universidad Nacional de Quilmes en Argentina. Este enfoque se centra en las potencialidades de cambio social que presenta el desarrollo de experiencias tecnológicas inclusivas, que deben contar con la participación de movimientos y organizaciones sociales desde su concepción.

En cuanto a la comprensión de las políticas CTI, existe un relativo acuerdo entre estas diversas posturas teóricas en las limitaciones de su concepción actual, que las restringe a objetivos de crecimiento y competitividad económica, inhibiendo su posible aporte a otras áreas del desarrollo. Sin embargo, hay diferencias en la profundidad y radicalidad de los cambios propuestos según el enfoque teórico, desde críticas frontales a la interpretación normativa de los principios de la economía de la innovación (enfoques CTS, principalmente el concepto de tecnología social), a una combinación 
de objetivos que permitan una complementación y maximización entre metas sociales y metas de crecimiento económico (enfoque de organismos internacionales).

Las experiencias recientes de los países de la región que han incorporado en el discurso de sus políticas de CTI la cuestión de la inclusión social constituyen un valioso campo de análisis, ya que permiten observar cómo se traducen en la práctica las distintas concepciones teóricas sobre el desarrollo Сті y sus vínculos con los procesos de exclusión/inclusión social.

\section{POLÍTICAS DE CIENCIA, TECNOLOGÍA E INCLUSIÓN SOCIAL EN BRASIL}

Luego de la victoria del Partido de los Trabajadores (РT) en las elecciones presidenciales de 2002, hubo una fuerte orientación por parte del gobierno, en especial por impulso del presidente Luiz Inácio Lula da Silva, para que todas las áreas formaran parte de la estrategia de combate al hambre y la pobreza (Bresser-Pereira, 2007).

El PT comienza a llevar a cabo políticas sociales y redistributivas importantes que estrechan sus vínculos con los sectores excluidos del mercado de trabajo (Sader, 2009: 97). Se implementó un modelo de mayor participación del Estado en la economía, planteándose una "nueva estrategia de desarrollo" que presentó algunas continuidades con la política económica del gobierno anterior de Fernando Henrique Cardoso; aportó, no obstante, rasgos distintivos en lo que respecta a la implementación de políticas industriales y de infraestructura, así como cambios en materia de política social.

De esta forma entran en la agenda política los temas de exclusión social. Su materialización se dio a través del programa Fome Zero (Hambre Cero, que luego pasaría a convertirse en estrategia gubernamental) y de las políticas de inclusión asociadas a este.

En el área de ciencia y tecnología, la orientación de combate al hambre y la pobreza se vio reflejada en la creación de nuevos espacios institucionales, en el destino de recursos y en la priorización de temas para la inclusión social (Fonseca, 2009: 14).

A pesar de la continuidad de algunos aspectos en la política económica del gobierno liberal de Fernando Henrique Cardoso, la introducción de cambios incrementales en las políticas sociales y de desarrollo generaron una alteración parcial en el pacto político que sustentaba al gobierno, produciendo una presión que permitió la introducción del tema de ciencia y tecnología para el desarrollo social en la PCTI brasilera (Fonseca, 2009: 8). 
Autores como Dagnino (2007), Fonseca (2009) y Dias (2012) consideran que la política de ciencia y tecnología para el desarrollo social (PCTDS) pudo ser creada debido a que entró en la agenda sin desequilibrar de manera relevante el balance de fuerzas existente, el contenido general y la orientación tradicional de la PCTI brasilera.

\section{El camino hacia la estrategia nacional de ciencia, tecnología e innovación}

El Plan Plurianual del Ministerio de Ciencia y Tecnología (PPA) 2004-2007 tuvo por objetivo inaugurar una estrategia de largo plazo basada en la inclusión social y la desconcentración de la renta con crecimiento (ambientalmente sustentable) del producto y del empleo (мСт, 2004).

En los años precedentes a la aprobación del Ppa se realizaron diversos debates entre parte de la comunidad de investigación, órganos estatales (Ministerio de Ciencia y Tecnología, мcт; Consejo Nacional de Desarrollo Científico y Tecnológico, Cnpq; Financiadora de Estudios y Proyectos, FINEP; Centro de Gestión y Estudios Estratégicos, CGEE) y ONG, articulados por la Academia Brasilera de Ciencias y por el Instituto de Tecnología Social (ITS), instituto del tercer sector que promociona la tecnología social y asistiva en Brasil. A partir de estos debates surgen dos grupos de trabajo; uno, Ciencia, Tecnología y Tercer Sector, tenía por objetivo elaborar propuestas de articulaciones entre el tercer sector y el мСт. El otro grupo creado fue Tecnología para el Desarrollo Social en noviembre de 2002, del cual surge la propuesta de creación de una secretaría del мст que actúe en los temas relacionados a la inclusión y el desarrollo social (Dias, 2012: 163).

La Secretaría Nacional de Ciencia y Tecnología para la Inclusión Social (secis) fue creada, en junio de 2003, como órgano responsable de "apoyar programas y proyectos que tengan por objetivo común la utilización de la ciencia, la tecnología y la innovación como puertas de acceso de la población más pobre del país a los beneficios del progreso" (МСт, 2007: 108). Surge por la concepción del gobierno federal de que no es posible el desarrollo sin inclusión social y por las presiones de los diversos actores vinculados al tercer sector, junto con los actores tradicionales de la PCTI brasilera (comunidad de investigación y burocracia estatal). Entre estos actores involucrados, el grupo de ONG que se articula y presiona en torno a la creación de la SECIS no participaba hasta entonces activamente de la elaboración de la PCTI. La articulación de actores públicos, del tercer sector, comunidad de investigadores y burocracia estatal, en el marco de esta política, constituye una novedad. De acuerdo a Dias (2012), la articulación entre actores inte- 
resados en influenciar la РCT se constituye en un paso fundamental en el sentido de reorientar el patrón de la política. La conquista de espacio en el juego político de esta nueva coalición de actores (comprometidos con las luchas sociales) debe ser entendida en el contexto de consolidación de las bases democráticas del país. Esos grupos habían sido silenciados por el autoritarismo en la dictadura militar y recién pasaron a conquistar espacios en el gobierno de Lula da Silva (Dias, 2012: 169).

Un actor relevante en las etapas iniciales de estas transformaciones es la Red de Tecnología Social (RTS), que nucleaba a un conjunto de ONG y movimientos sociales vinculados con experiencias de tecnologías para la inclusión social en distintos estados del país. El proceso que llevó a la creación de la RTS tiene varios orígenes, entre los cuales pueden marcarse la creación del Instituto de Tecnología Social en el 2000 por parte de las ong y la creación de la Fundación del Banco de Brasil (FвB) por parte del gobierno (Fonseca, 2009). La creación de la RTS comenzó a ser debatida por distintos actores que luego la integraron en el marco de la segunda edición del Premio Fundación Banco de Brasil de Tecnología Social (lanzado por la FBB) en 2003. Allí, a partir del impulso del gobierno, se promovieron reuniones con diferentes instituciones, estimulando el debate y la articulación de los actores sociales (Fonseca, 2009). En un primer momento participaron de la RTs: la FBB, el ITs, la SECIS/MCT, la FINEP, el Centro Avanzado de Tecnologías Sociales del Instituto Ayrton Senna, la empresa Petróleo Brasilero S.A (Petrobras) y el Servicio Brasilero de Apoyo a las Pequeñas y Medianas Empresas (SEBRAE).

En aquel momento el мCT definió, como objetivo de la Política de Ciencia y Tecnología para el Desarrollo Social (PCTDS), lo siguiente:

Identificar, articular y apoyar las demandas locales, territoriales y regionales de tecnologías sociales, con el objetivo de apoyar y financiar la investigación, innovación y extensión que contribuya para la inclusión social de las poblaciones más vulnerables a la pobreza en el proceso de desarrollo y reducción de las desigualdades regionales (мСт, 2007: 58).

En el momento de creación de la PCTDs (2003) no hubo una elaboración explícita de una política o un plan; sin embargo, es posible identificar lo que fue la política a través de los programas y proyectos implementados y los discursos sobre el tema. Recién en el año 2004, con el Plan Plurianual 2004-2007aparece un plan de acción estructurado en la materia. Esto se debe a que los planes plurianuales son elaborados en el primer año de gobierno para los cuatro ańos siguientes; por tanto, el primer plan del 
gobierno de Lula da Silva fue elaborado durante el 2003 para su ejecución entre 2004 y 2007 (Fonseca, 2009: 16).

Es importante resaltar que para la elaboración de este plan de acción y de los siguientes (PPA 2007-2010 y ENCTI 2012-2015) se utilizó la herramienta participativa de las conferencias nacionales de cTr. Esta herramienta no surge en el período analizado, sino que la primera edición fue en 1985, cuando se crea el мст, y la segunda en 2001, durante el gobierno de Fernando Henrique Cardoso (cuando comienza a promoverse la participación de las ONG y organizaciones sociales en el proceso decisorio). La herramienta es retomada y modificada por los gobiernos de Lula da Silva: se celebran la tercera y la cuarta Conferencia, en 2005 y 2010 respectivamente, con el fin de ofrecer a la sociedad un espacio democrático donde manifestarse sobre propuestas y aspiraciones para el sector (Livro Azul, 2010).

Estas conferencias y sus instancias preparatorias permitieron la participación de diversos actores sociales en la discusión de temas y prioridades de la PCTI en Brasil; por tanto, constituyeron un importante aporte para ampliar la concepción de la importancia de la cTi para el desarrollo del país y propiciar el involucramiento de otros actores organizados de la sociedad civil a la discusión sobre estos temas. Sin embargo, la comunidad académica siguió representando la mayoría de participantes en los referidos debates (Arbix, 2010; Serafim y Dagnino, 2011; Pereira y Escada, 2012).

Si bien la participación de organizaciones sociales a partir de la segunda Conferencia Nacional de Ciencia y Tecnología se consideró un paso adelante, fue incipiente y restringida a la lógica ofertista lineal de сті (Serafim y Dagnino, 2011). Un ejemplo de esto es que gran parte de las orientaciones de los ejes de la ENCTI fue determinada por el Consejo Nacional de Ciencia y Tecnología (ССT) ${ }^{[2]}$ que estuvo compuesto por cuatro comisiones específicas (una por cada eje estructurante de la ENCTI) en las que existió escasa presencia de las organizaciones de la sociedad civil vinculadas a estos temas. En el caso del último eje, el referido al tema de este trabajo: comisión de acompańamiento en CYT para el desarrollo social, estuvo coordinado por el presidente del Forum de Secretarios Municipales de CYT y un representante de la ASGA. ${ }^{[3]}$ Además, contaba con dos representantes del Consejo Nacional de Secretarios Estaduales para asuntos de ciencia, tecno-

[2] Órgano consultivo de asesoramiento superior del presidente de la República para la formulación e implementación de la política nacional de ciencia, tecnología e innovación, creado por ley $\mathrm{N}^{\circ}$ 9.257, el 9 de enero de 1996.

[3] ASGA, empresa brasilera de desarrollo de tecnologías de comunicación por fibra óptica y transmisión digital, fundada en 1989. 
logía e innovación (CONSECTI), ${ }^{[4]}$ por la Confederación Nacional de las Industrias (CNI), representantes del Forum de Secretarios Municipales de CYT, ${ }^{[5]}$ por la Empresa Brasilera de Investigación Agropecuaria (EMBRAPA) ${ }^{[6]}$ y por el Ministerio de Salud. No existió representación de organizaciones de la sociedad civil.

Pereira y Escada (2012) destacan la participación del Instituto de Tecnología Social, la Red de Tecnología Social, la Fundación Banco de Brasil y otras organizaciones no gubernamentales en las conferencias nacionales, para impulsar una PCTI orientada al desarrollo social. Sin embargo, se vislumbra una desigualdad en el acceso al poder decisorio en la PCTI, con hegemonía de concepciones basadas en la universalidad y neutralidad de la ciencia, detentadas por la comunidad académica (Serafim y Dagnino, 2011). Esta comunidad mantuvo desde la institucionalización de la PCTI brasilera, en la década de 1950, el control sobre la agenda, siendo el actor dominante a lo largo de toda su trayectoria. La burocracia estatal fue perdiendo fuerza como actor en estas políticas desde la década de 1980 por las políticas neoliberales hacia la estructura estatal; las empresas también pierden fuerza como actor influyente en la política a partir de esa época. Mientras la comunidad académica mantiene su incidencia, y aun hegemonía, las demandas de movimientos y organizaciones sociales están poco representadas o no tienen fuerza suficiente para ocupar una posición privilegiada en el proceso de toma de decisiones y en la propia agenda de la PCTI. Los resultados de esas instancias decisorias se reflejan claramente en los planes y en la creación de una secretaría (secis) con acciones difusas y de poco peso en el marco de los lineamientos de la рсті general (Dias, 2012: 173).

La Red de Tecnología Social, formada en 2004 con cerca de 900 actores entre organizaciones de la sociedad civil, instituciones académicas, organismos gubernamentales y empresas públicas, es un ejemplo inédito de alianza para la reorientación de la agenda de ciencia y tecnología de un país latinoamericano hacia fines sociales y de fuerte participación de la sociedad civil

[4] CONSECTI, entidad privada sin fines lucrativos fundada en 2005 con la finalidad de representar a las secretarias de Ciencia y Tecnología.

[5] Conformado en 2001 es un órgano que intenta articular las secretarias municipales y establecer relaciones con órganos del gobierno estadual y federal en relación a los temas de ciencia, tecnología e innovación.

[6] embrapa, institución estatal federal pública brasilera vinculada al Ministerio de Agricultura, Ganadería y Abastecimiento fundada en 1973. Sus objetivos son desarrollar tecnologías, conocimiento e información técnicas y científicas para la agricultura y la ganadería brasilera. 
organizada, ${ }^{[7]}$ con presencia en las conferencias nacionales para la elaboración de la encti (Serafim y Dagnino, 2011; Pereira y Escada, 2012).

\section{El plan de acción en ciencia, tecnología e innovación 2007-2010}

En 2007, durante el segundo gobierno de Lula da Silva, se lanzó un Plan de acción para el período 2007-2010, "Ciencia, Tecnología e Innovación para el Desarrollo Nacional”, subordinado al Plan Plurianual del gobierno federal (PPA); su elaboración estuvo orientada por las conclusiones de la III Conferencia Nacional de Ciencia y Tecnología (мст, 2007).

Este plan marcaba la importancia de que la producción de conocimiento científico-tecnológico estuviera al servicio de una estrategia más amplia orientada al desarrollo económico y la solución de los problemas sociales del país. Para llevar a cabo este objetivo, el plan se estructuraba en cuatro ejes orientadores: uno horizontal, de carácter estructural, y tres ejes verticales. El primero apuntaba a consolidar el sistema nacional de ciencia, tecnología e innovación, promoviendo la mejora de la infraestructura, el fomento a la investigación y la formación de recursos humanos. Los ejes verticales eran: promoción de la innovación tecnológica en las empresas; investigación, desarrollo e innovación en áreas estratégicas y ciencia, tecnología e innovación para el desarrollo social (Dias, 2012: 146).

El primer eje establecía un vínculo entre las actividades de cTi y las prioridades de la política industrial, tecnológica y de comercio exterior (PITCE), lanzada en 2004 por el gobierno federal con el objetivo de fortalecer la base industrial brasilera. El segundo eje vertical tenía la finalidad de concretar los objetivos estratégicos nacionales por medio de programas que apuntaban a la soberanía del país en las áreas espacial, nuclear y las ligadas a la Amazonia. El tercer eje apuntaba a estimular la inclusión y el desarrollo social con el apoyo de ciencia, tecnología e innovación (Dias, 2012: 147).

La IV Conferencia Nacional de cYT fue el núcleo del debate público organizado por el gobierno luego de finalizado el Plan de Acción en CTI en 2010. Dicho debate sobre las prioridades nacionales en la materia, surgió a partir del análisis de necesidades del sector identificadas por un grupo de expertos coordinado por el Ministerio de Ciencia y Tecnología, con la idea de dar continuidad y profundidad a las acciones establecidas en el Plan de

[7] Del total de organizaciones que integraron la RTs, más de 500 eran organizaciones sociales o no gubernamentales y más de 100 instituciones privadas. La financiación provenía de diversos organismos públicos (RTS, 2011:3). 
Acción (IV CNCT). La IV Conferencia se realizó en mayo de 2010 en Brasilia con una duración de tres días, fue precedido por varias reuniones estatales y municipales, además de foros de discusión en todo el país que sirvieron como espacios para identificar las principales cuestiones y temas abordados en el evento de nivel nacional. En dichos espacios participaron, además de representantes de la academia y organismos estatales, organizaciones de la sociedad civil y los movimientos sociales, fundamentalmente agrupados en la RTS e instituciones no gubernamentales como el Instituto de Tecnología Social. Ese proceso culminó con la elaboración de la Estrategia Nacional de Ciencia, Tecnología e Innovación (ENCTI) 2012-2015, que fue aprobada en 2012 y se basó en el Plan de acción 2007-2010.

Siguiendo a Pereira y Escada (2012: b52), quienes realizaron una investigación mediante la utilización de técnicas de observación participante, estas conferencias (realizadas a nivel estadual, regional del nordeste y nacional) si bien habilitaron la participación de nuevos actores de la sociedad civil organizada en los aspectos de ciencia y tecnología para el desarrollo, siguieron en mayor medida un modelo tradicional de alfabetización científica de los ciudadanos, reservando un lugar privilegiado a los especialistas que informan al público sobre los resultados y potencialidades de la CTI, con escasa apertura a recoger opiniones y demandas de los participantes para la definición de la política.

\section{La Estrategia Nacional de Ciencia, Tecnología e Innovación (2012-2015)}

La Estrategia Nacional cті (2012-2015) mencionaba, como principales desafíos a ser enfrentados en el período, la reducción del desfasaje científico y tecnológico que separa a Brasil de los países más desarrollados; la expansión y consolidación del liderazgo brasilero en la economía del conocimiento de la naturaleza; la ampliación de las bases para la sustentabilidad ambiental y el desarrollo de una economía de bajo carbono; la consolidación del nuevo modelo de inserción internacional del Brasil; y la superación de la pobreza y la reducción de las desigualdades sociales y regionales (MCTI, 2012: 33-39).

La Cті para el Desarrollo Social -que incluía la popularización de la Сті, la mejora de la enseñanza de las ciencias, la inclusión productiva y social y las tecnologías para las ciudades sustentables- fue planteada como uno de los sectores portadores de futuro para los que se establecían programas prioritarios. Entre ellos también se encontraban: tecnologías de la información y la comunicación, fármacos y complejo industrial de salud, 
petróleo y gas, complejo industrial de defensa aeroespacial y nuclear, innovación en biotecnología y nanotecnología, economía verde en lo que refiere a energía renovable, biodiversidad y cambio climático (мСті, 2012: 54-86).

En сті para el Desarrollo Social estaban previstos los siguientes subgrupos de programas, dentro de Tecnologías para la Inclusión Social: programa de innovación en tecnología asistiva, inclusión digital, centros vocacionales tecnológicos y mejora de la gestión (мСті, 2012: 110-112).

Dos apreciaciones importantes surgen en este punto: primero, que los programas que se incluyen dentro de Tecnologías para Inclusión Social refieren en gran parte a cuestiones vinculadas con la inclusión digital (centros vocacionales tecnológicos y mejora de la gestión) y con la inclusión de personas en situación de discapacidad (tecnología asistiva).

Segundo, las prioridades de la política explícita siguen estando fuertemente vinculadas a la mejora de la competitividad y las metas económicas, quedando subordinados a estas los aportes que la сті puede realizar a la inclusión y desarrollo social. Además, estos aportes quedan expresados, la mayor parte de las veces, en términos de ampliación del acceso por parte de la población a nuevos conocimientos y tecnologías, quedando la noción de inclusión social relacionada a CTI vinculada a las ideas de "transferencia", "diseminación" y "acceso" al conocimiento.

Por otro lado, analizando la concepción del vínculo entre desarrollo cTI e inclusión y desarrollo social en el documento, parecería primar un enfoque determinista tecnológico de estos procesos, en el cual se le asigna un rol central al desarrollo científico y tecnológico como responsable y capaz de promover por sí solo la inclusión y el desarrollo social. Este concepto se basa en la idea de que la tecnología es el motor de los cambios sociales, económicos e históricos (Dagnino, 2008: 31), respondiendo al imaginario colectivo de un amplio sector de la población de que la ciencia y la tecnología son liberadoras en sí mismas y que el desarrollo científico-tecnológico se presenta como una "mejoría real, inexorable y efectiva en todos los aspectos de la vida humana” (Bazzo, 2011: 93). En este sentido, en la ENCTI se afirma que:

Siendo la innovación y el conocimiento los motores de la política de desarrollo, es esencial que la diseminación de nuevos conocimientos y nuevas tecnologías y métodos sean capaces de ampliar el acceso de la población a nuevos bienes y servicios, y que generen mejorías concretas para la colectividad, disminuyendo las desigualdades sociales existentes (ЕNCTI, мCTI, 2011: 28). 
Estas concepciones de supremacía de la ciencia y la tecnología en su articulación con la inclusión social se refuerzan a lo largo de todo el documento, haciendo énfasis en los efectos transversales de la cTI en la actividad económica, la conservación de los recursos naturales y a su capacidad de elevar los patrones de vida de la población brasilera a partir de su creciente incorporación al proceso productivo (MCTI, 2011: 33).

\section{Instrumentos, programas y presupuesto en la PCTDS (2004-2010)}

En el período analizado se encuentra una diversidad de programas e instrumentos dentro de la Secretaría de Ciencia, Tecnología e Innovación para la Inclusión Social (sECIs) del Ministerio, divididos en dos grandes áreas: Popularización de la CTI y Desarrollo de Tecnologías para la inclusión social.

En el período 2004-2010, que va del comienzo de la PCTDs hasta la finalización del Plan de Acción 2007-2010, en la primer área mencionada se abren cuatro programas y en la segunda ocho, que contaron con una relativa continuidad. Este período es el único para el cual se encontraron datos de presupuesto invertido en las diversas fuentes consultadas, por tanto se tomara como referencia para el análisis comparativo.

La diversidad de programas en el MCTI brasilero puede estar relacionada con la existencia de una multiplicidad de actores sociales y políticos con concepciones diferentes sobre exclusión/inclusión social, presentes en la disputa de poder de la рст brasilera, tal como señala Dias (2012), que llevan a la creación de instrumentos diferentes con diversas orientaciones.

Se incluyen dentro de esos programas aspectos agropecuarios, de desarrollo local y regional, de inclusión digital, de seguridad alimentaria, de ciudadanía y de discapacidad, entre otros. Esta imprecisión en relación con los instrumentos aplicados lleva a perjudicar la concreción del objetivo mayor de creación y difusión de conocimientos para el desarrollo social, diluyendo esfuerzos en otros temas que no corresponden directamente con el objetivo o que ya son atendidos por otros programas y fondos en la órbita de otros ministerios (es el caso claro de las acciones de inclusión digital, ejecutadas en varios ministerios simultáneamente y sin coordinación) (Dias, 2012).

En cuanto al presupuesto ejecutado, tomando como base un estudio realizado por Pimentel y Cavalcante (2012), en el período 2003-2010 el total de recursos destinados al tercer eje vertical de los cuatro del MCTI, el que se relaciona con los objetivos de este trabajo representa el $5.6 \%$ del total del presupuesto del мсті para el período (1.075 de 19.059 millones de 
dólares de 2010). Esto muestra la baja prioridad asignada a estos temas en el presupuesto nacional de CTI.

Se observa una importante fluctuación en el presupuesto asignado en cada año para esta política, registrándose el punto más alto en 2005 con una inversión que corresponde al 9,2\% del total ejecutado por el мсті y el punto más bajo en el año 2003, representando el 0,2\% del presupuesto total.

La situación analizada hasta este punto se vio modificada en los últimos años, a partir de la asunción en 2016 del presidente Michel Temer, con la decisión de fusionar el Ministerio de Ciencia, Tecnología e Innovación con el de Comunicaciones y reunir a la Secretaria de Ciência e Tecnologia para Inclusão Social (sECIs) con la Secretaria de Políticas e Programas de Pesquisa e Desenvolvimento (SEPED). Según Natal y Alvim (2018: 84), a partir de esta fusión, entre otros aspectos, se dejó de priorizar la popularización y difusión de la CTI y de relacionarla con la inclusión social. Aunque no hay demasiada información rigurosa al respecto, estas acciones de fusión de estructuras parecen implicar un cierto retroceso en las políticas de сті en general, y en particular en las vinculadas a inclusión social.

\section{Figura 1. Presupuesto ejecutado en MCт por eje de actuación (en millones de dólares)}

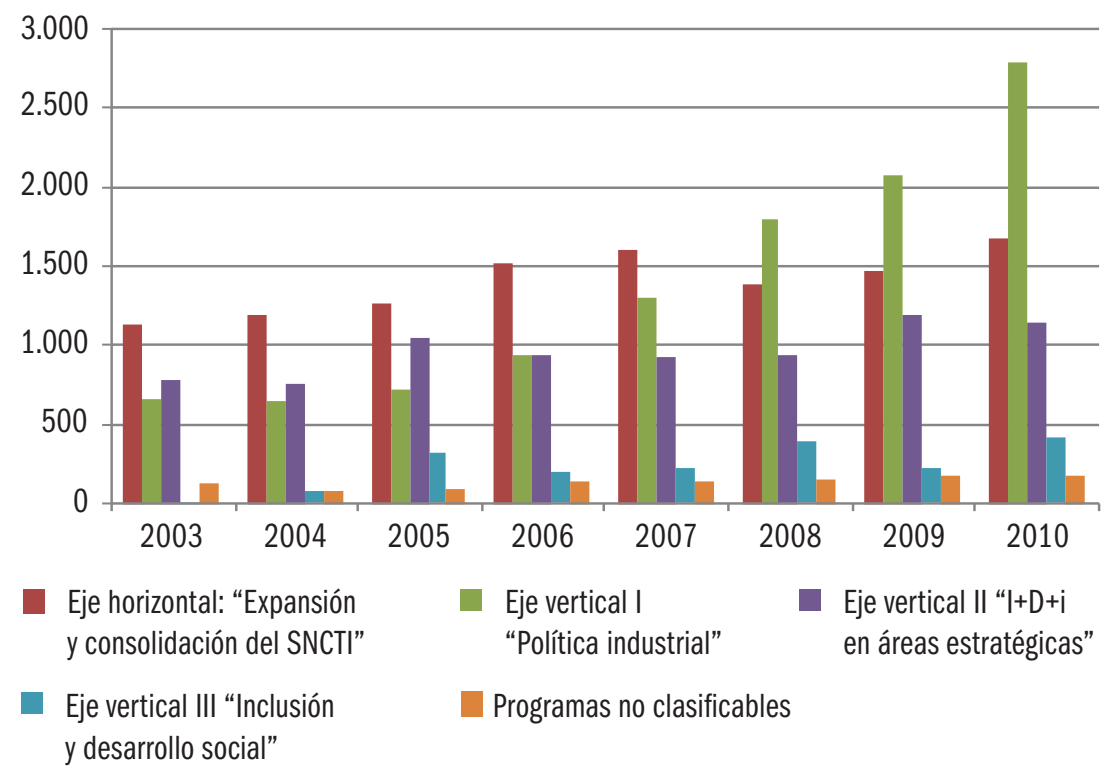

Fuente: Elaboración propia basada en datos de Pimentel y Cavalcante (2012). 


\section{EL CAMINO HACIA UNA POLÍTICA DE ESTADO EN CTI EN URUGUAY}

Previo a la asunción del primer gobierno progresista, en el año 2003, diferentes actores, tanto políticos como académicos, comenzaron a discutir el tema del desarrollo científico y tecnológico del país. La crisis económicosocial de 2002 golpeó duramente a esta área, paralizando los trabajos de investigación y retrayendo los avances realizados en materia de innovación empresarial, que se había intentado promover a partir de fines de la década de 1980. Esto llevó al desarrollo de una creciente preocupación por parte de los actores políticos respecto a CTI; se creó una comisión del Senado sobre el tema, el Parlamento convocó a instancias de discusión, se creó el denominado proyecto CIENTIS en 2003 (que tuvo como objetivo elaborar un programa de desarrollo para СтI), se redactaron varios anteproyectos de ley y todos los partidos políticos comenzaron a incorporar el tema en sus plataformas electorales (Rubianes, 2009: 194-195).

\section{Del proyecto cIENTIS a consultorías contratadas para un Plan}

El proyecto CiENTIS se desarrolló durante 2003 y constituyó el primer antecedente de diagnóstico y propuestas políticas para el campo de la ciencia, tecnología, innovación y sociedad. Fue convocado por un conjunto de actores privados - casi 300 participantes, incluyendo académicos, políticos y especialistas en la temática- y auspiciado por la Fundación Friedrich Ebert en el Uruguay (FESUR). El documento en el cual culminó el proyecto menciona una serie de problemas y potencialidades que constituyen el punto de partida, en la época, en ciencia y tecnología, además de la elaboración de criterios y aportes para la creación de una política nacional de ciencia y tecnología.

A partir de esta experiencia, se comienzan a consolidar en Uruguay transformaciones conceptuales que se venían dando con mayor fuerza desde la década de 1980 a nivel mundial: se desarrolla, a nivel discursivo, un enfoque de CTI asociado a modelos sistémicos, interactivos y con participación de actores sociales, en un intento de superación de los enfoques lineales.

Otro antecedente de discusión fue un estudio sobre la situación y perspectivas de la Сті en Uruguay, realizado por un grupo de investigadores del campo ciencia, tecnología, sociedad y desarrollo en 2004, financiado por el BID. De él se derivó una publicación más extensa y detallada de la Universidad de la República, "Ciencia, tecnología e innovación en Uruguay: diagnóstico, prospectiva y políticas” (Bértola et al., 2005). 
A partir de estos documentos, el Gabinete Ministerial de la Innovación $(\mathrm{GMI})^{[8]}$ planteó las bases, punto de partida y algunos lineamientos estratégicos que pretendieron servir de referencia para la discusión entre los diversos agentes, tanto públicos como privados, vinculados a CTI, con la finalidad de llegar a acuerdos y consensos en torno al contenido de un Plan Estratégico Nacional que se elaboraría a partir de este proceso. Este documento, publicado en 2007 bajo el nombre Bases y principales lineamientos del PENCTI, plantea como premisa un modelo de desarrollo basado en el conocimiento, en el cual la innovación entendida como la creación y uso de nuevos conocimientos, productos, procesos, bienes, servicios, herramientas y formas de organización, cumple un rol central.

La innovación aparece fuertemente vinculada, en las visiones del GMI, con la creación de capacidades para la competitividad de las empresas y la generación de las condiciones adecuadas para ello. La inclusión social es introducida como parte de estas condiciones necesarias para que la competitividad internacional, que "demanda el mundo moderno", pueda llevarse a cabo y sostenerse en el tiempo. Como último elemento de la estrategia de fomento a la CTI es planteada "la integración social ante el riesgo cierto de seguir agudizando los procesos sociales de exclusión vinculados a las nuevas modalidades que imprime la irrupción de las nuevas tecnologías sobre el mundo del trabajo y la convivencia social" (GMI, 2007: 4).

A partir de la difusión de los lineamientos básicos para la discusión del PENCTI por parte del Gabinete Ministerial en diciembre de 2007, la Universidad de la República aprobó un documento que intentaba dar respuesta a esa propuesta. Allí se plantea, como una de las principales sugerencias, la necesidad de realizar diagnósticos y de utilizar el conocimiento acumulado por los especialistas en las diferentes temáticas para elaborar un Plan Nacional de Ciencia y Tecnología acorde con los problemas y necesidades del país.

Tomando en consideración estos comentarios, el Gabinete encomendó la contratación de nueve consultorías que fueron desarrolladas durante el período 2008-2009 por técnicos de reconocida capacidad. Siete de ellas corresponden a sectores prioritarios establecidos para fomentar la investigación e innovación (agroindustrial, energético, biotecnológico, salud, turismo, medio ambiente, tecnologías de la información y comunicación)

[8] Creado en 2005, integrado por el director de la Oficina de Planeamiento y Presupuesto y los ministros de Educación y Cultura; Economía y Finanzas; Ganadería, Agricultura y Pesca; e Industria, Energía y Minería, debía liderar el proceso de construcción de políticas públicas en investigación e innovación. 
y las dos restantes fueron de corte sistémico: redes de innovación públicoprivadas e innovación e inclusión social. Además de estas consultorías, se contrató un estudio con el fin de sintetizar el conjunto de los aportes sectoriales en un único texto (GMI, 2010).

Se puede destacar que el tema de la innovación para la inclusión social es planteado no como un área específica más de intervención, sino como un componente transversal del plan en elaboración. Esta perspectiva se ve ampliamente reflejada en la consultoría que sintetiza y profundiza el conjunto de aportes de las consultorías sectoriales, "Ciencia, tecnología e innovación en Uruguay" finalizada en abril de 2009, cuyo informe final se convierte en el documento base para la estructura y propuestas que contendrá el PENCTI aprobado luego.

En el primer capítulo del informe se presenta su perspectiva global, y una de las premisas fundamentales es "partir de una concepción integral del desarrollo en la que la equidad y el desarrollo son elementos de una misma moneda". Se alude a la necesidad del aporte de la investigación e innovación al crecimiento con equidad. Esto implica la combinación e interacción de políticas macroeconómicas, políticas de desarrollo productivo y políticas sociales orientadas a la eliminación de diversas formas de discriminación mediante inversión en capital humano y construcción de capital social (Bianchi y Snoeck, 2009: 6).

Luego se establece el marco conceptual en el que se apoyará el PENCTI, planteando dos enfoques complementarios: a) Enfoque macro: integración dinámica entre sectores económicos: consiste en "abarcar las capacidades de innovación en el sistema productivo como un todo, de manera de potenciar las ventajas de complementariedad y los procesos de sinergia"; plantea el concepto de Sistema Nacional de Innovación como base, entendiendo que la interacción entre sectores y los diferentes actores que intervienen en su desarrollo "genera complementariedades y externalidades positivas que son esenciales para mejorar la productividad y la competitividad de una economía"; b) Enfoque social: el aporte a la equidad social desde la investigación: se plantea la idea de contribuir a resolver los problemas de pobreza y exclusión social desde las actividades de CTI, partiendo de reconocer que la intensificación en conocimiento de la economía no acarrea automáticamente una reducción de la desigualdad, sino que muchas veces se produce el efecto contrario, exacerbándose mediante estos avances la polarización social y la inequidad (Bianchi y Snoeck, 2009: 12-15).

La centralidad dada a la vinculación entre el desarrollo científico-tecnológico y los procesos de inclusión/exclusión social queda evidenciada a lo largo del documento y principalmente en su marco conceptual de base, 
lineamientos estratégicos y objetivos principales. El tercer objetivo general de la propuesta refiere directamente a esta temática, "Desarrollar capacidades y oportunidades para la apropiación social del conocimiento y la innovación 'inclusiva". A su vez, este objetivo se divide en tres subobjetivos; a) la generación y aplicación de conocimientos para la resolución de problemas sociales y la inclusión social, b) la divulgación de los avances científico-tecnológicos favoreciendo la apropiación social del conocimiento por parte de los ciudadanos y c) el fomento del espíritu emprendedor de niños y jóvenes mediante programas de popularización de la ciencia y tecnología.

La importancia asignada a la divulgación y popularización de CYT dentro de este objetivo es notoria, está presente en dos de los tres subobjetivos planteados. Sin embargo, cuando se desarrolla este punto en el capítulo referido a "Desafíos estratégicos a nivel sectorial", se le asigna mayor relevancia a los aspectos referidos a la contribución del PENCTI a la resolución de problemas sociales mediante la generación de conocimiento endógeno.

El enfoque en el que se basan los objetivos de política desarrollados en este documento sobre la relación entre CTI y equidad social intenta superar la visión orientada únicamente a la reducción de los impactos negativos de los cambios tecnológicos, mediante la promoción de políticas orientadas a aprovechar las capacidades de investigación e innovación para la resolución de problemas de equidad social, haciendo énfasis en la vinculación entre las demandas surgidas de los problemas presentes en la vida cotidiana y las soluciones que pueden brindarse desde la producción de conocimiento científico-tecnológico (Sutz, 2008).

\section{El Plan Estratégico Nacional de cTI 2010-2030}

El largo proceso de discusión y elaboración de propuestas en torno al rumbo de la Cті en Uruguay culmina con la aprobación, mediante decreto presidencial del 27/02/2010, del Plan Estratégico Nacional de Ciencia, Tecnología e Innovación para el período 2010-2030.

El documento aprobado parece, en principio, una versión resumida del informe final de consultoría realizado en 2009. Sin embargo, cuando se refiere a áreas estratégicas prioritarias hay algunas diferencias. Se establece una distinción entre sectores productivos y sociales, áreas tecnológicas intensivas en conocimiento y desarrollo de capacidades de base. Marca tres niveles de priorización: el primero involucra a sectores "intensivos en conocimiento" o "tecnologías transversales", el segundo refiere a la formación y 
desarrollo de capacidades de base, y el tercero son los núcleos de problemas relacionados con sectores productivos y sociales.

A lo largo del texto del Plan no se encuentra ninguna alusión a la inclusión social como área de problemas a abordar, a diferencia de la consultoría previa. Al igual que en el informe final de consultoría, el tema aparece como el tercero de los cinco objetivos del PENCTI ${ }^{[9]}$ ("desarrollar capacidades y oportunidades para la apropiación social del conocimiento y la innovación "inclusiva"), pero en este caso se sustituyen los problemas referidos a la inclusión social por el área de problemas de "educación y desarrollo social", estableciendo un carácter más amplio a la categoría y al tipo de problemas que ella puede incluir.

Los principales actores que participaron en el proceso de discusión y elaboración del plan fueron actores gubernamentales, académicos y asesores técnicos de organismos internacionales, fundamentalmente del BID. Este aspecto, sin dudas, imprimió características particulares al documento. La asesoría y cooperación técnica de este organismo tuvo fuerte relevancia en el proceso debido a que la elaboración del plan se enmarca en una serie de transformaciones institucionales de la PCTI incluidas bajo el eje estratégico "Uruguay Innovador" del gobierno de Tabaré Vázquez que fueron financiadas en su mayoría por préstamos de dicho organismo.

Luego de las consultorías temáticas contratadas, se realizaron talleres para discutir los resultados de cada tema. En el área de inclusión social el taller de discusión de resultados estuvo a cargo de los especialistas en ciencias sociales que realizaron la consultoría y asistieron alrededor de veinte expertos académicos sin registrarse participación de otros actores sociales.

Es pertinente mencionar que el Plan Estratégico es de carácter muy general, no llegando al planteo de acciones y herramientas específicas mediante las cuales sea posible alcanzar los objetivos propuestos para cada sector. Esto deja un margen de acción muy amplio para las instituciones encargadas de la ejecución de los programas, proyectos y acciones tendientes a la concreción práctica del Plan.

[9] Obj. 1: Consolidar el sistema científico y tecnológico y su vinculación con la realidad productiva y social. Obj. 2: Incrementar la competitividad de los sectores productivos en el escenario de la globalización. Obj. 3: Desarrollar capacidades y oportunidades para la apropiación social del conocimiento y la innovación "inclusiva”. Obj. 4: Formar y capacitar los recursos humanos requeridos. Obj. 5: Desarrollar un sistema de prospectiva, vigilancia y evaluación tecnológica y de evaluación de políticas públicas e instrumentos de CTI (GMI/ PENCTI, 2010: 10). 


\section{Instrumentos y presupuesto ejecutado en el marco del PENCTI para inclusión y desarrollo social}

En la Agencia de Investigación e Innovación (ANII) uruguaya para el período 2010-2015, se desarrollaron tres instrumentos relacionados con la ejecución del pencti: Programas de Investigación de Alto Impacto Social, Fondo de Inclusión social y Popularización de la Ciencia y la Tecnología. Desde 2011 hasta finalizar el período analizado solamente hubo convocatorias y presupuesto permanente para el instrumento de Popularización de la CYT; los restantes fueron esporádicos, con mayor regularidad el de Alto Impacto Social (2008-2010), mientras que el de Inclusión Social, orientado en realidad a la discapacidad física y mental -que podría ser vinculado a otras áreas de intervención del Estado- contó con una única edición en 2009 donde se financiaron solamente cuatro experiencias y no se volvió a abrir una nueva convocatoria desde esa fecha. En 2016, luego de seis años de discontinuidad del instrumento de Alto impacto Social, se realizó una convocatoria a un nuevo instrumento, "Proyectos de Innovación Inclusiva” (ININ) que presenta objetivos muy similares.

Existen otras acciones a nivel estatal que abordan temas vinculados a la CTI para la inclusión social, como desarrollo tecnológico para la agricultura familiar o inclusión digital, pero no se encuentran bajo la órbita de la ANII como agencia estatal ejecutora del PENCTI, ni se relacionan con sus objetivos. Esto denota una imprecisión y falta de articulación y coordinación a nivel Estatal de instrumentos diversos relacionados con la temática.

Tal como se observa en la figura 2, la inversión en instrumentos de inclusión social tiene su punto más alto en el año 2009, ascendiendo al $4,4 \%$ del total ejecutado por la ANII en ese año. Esta cifra se debe a la implementación simultánea de tres instrumentos: Alto Impacto Social, Fondo de Inclusión social y Popularización de la cTi. El resto de los años la inversión no llega al $3 \%$ y en la mayoría no alcanza el $1 \%$.

\section{SIMILITUDES Y DIFERENCIAS ENTRE PAÍSES}

Se analiza a continuación las principales similitudes y diferencias entre la experiencia brasilera y la uruguaya en cuanto a la elaboración de planes de PCTI, en particular a la vinculación entre CTI e inclusión social y su inserción en la agenda de la PCTI, así como los programas e instrumentos desarrollados y sus presupuestos. 
Figura 2. Porcentaje asignado por programa ANII (2008-2016)



Fuente: Elaboración propia basada en la información disponible en <https://www.anii.org.uy/institucional/ documentos-de-interes/>.

La primera de las similitudes encontradas es que el impulso para la instalación de los temas de inclusión social en la agenda de la PCTI de ambos países estuvo enmarcado en sus respectivos gobiernos progresistas. La problematización realizada por los gobiernos de Lula da Silva, desde 2003, y Tabaré Vázquez, desde 2005, que identifica a los fenómenos de exclusión social y desigualdad como un problema público que requiere una intervención inmediata, en el marco de una agenda política más amplia tanto del Partido de los Trabajadores (Рт) como del Frente Amplio (FA), fue fundamental en ambos casos para que fuera posible su inserción en la agenda política de la PCTI de estos temas.

Este escenario político se produce en el marco del denominado "giro a la izquierda" en América Latina, que responde al fracaso de las reformas neoliberales aplicadas en la región en la década de 1990 a partir del Consenso de Washington. Estas reformas, lejos de lograr propiciar mayores niveles de desarrollo, se convirtieron en factores que profundizaron la dinámica de la crisis y empeoraron los problemas de exclusión social y pobreza en los países sudamericanos que las implementaron (Lechini et al., 2008).

Las nuevas autoridades gubernamentales consideraron la conveniencia que propiciaba este nuevo contexto político para plantear objetivos de desarrollo e inclusión social en la PCTI, haciendo coincidir el discurso de estas políticas con los planteamientos de índole social que eran la base de los programas de estos gobiernos (Arancibia, 2017: 132). 
Otro aspecto a considerar en la inserción de estos temas en las agendas gubernamentales son los procesos que se llevaron a cabo para la elaboración de los planes nacionales en ambos países. Considerando la relevancia de la participación social para generar procesos de inclusión y cohesión, se entiende fundamental la presencia de procesos participativos y deliberativos en la toma de decisiones sobre estos temas. En los dos países analizados se constata un acercamiento a procesos participativos en la elaboración de los planes nacionales de CTI, abriéndose espacios de consulta y participación.

Se constata como característica compartida en ambos países los mecanismos consultivos moderados en la elaboración de planes y programas que no incluyen de forma protagónica a otros actores "no tradicionales" de la рСт. Sin embargo, en el caso de Brasil se constató una relativa apertura a la incorporación de nuevos actores (aunque de forma un poco marginal) tales como organizaciones no gubernamentales (ONG) y movimientos sociales, proceso que no se registró en Uruguay.

Es pertinente comparar las características y el grado de prioridad que adquiere la PCTI para la inclusión social dentro de los planes o estrategias generales para el área, fruto de los procesos de discusión y elaboración antes mencionados. En el caso de Brasil encontramos, en el Plan de Acción 2007-2010, que la cTi para el desarrollo social es establecida como una de las cuatro prioridades estratégicas a nivel nacional (Prioridad Estratégica IV). Si bien se establece una orientación hacia la enseñanza de ciencias y la popularización de la CTI, también se incluyen los aspectos de producción y distribución de tecnologías e innovaciones orientadas a las problemáticas sociales. Así se conforman las dos líneas de acción propuestas para llevar a cabo este objetivo: Popularización de la cTi y mejora de la Enseñanza de Ciencias, por un lado, y Tecnologías para el desarrollo social por otro.

Se observa, sin embargo, prioridad asignada a tres temas considerados como centrales en la Política de сті para el Desarrollo Social: Centros Vocacionales Tecnológicos, Inclusión digital y Difusión y Popularización de la Ciencia. Queda en un segundo plano la producción tecnológica orientada a solucionar problemas de inclusión y desarrollo social, no incluida específicamente dentro de las metas, pero sí en las líneas de acción más generales.

Por tanto, no se percibe un cambio estructural de orientación del paradigma vigente de la PCTI, ya que los temas que implicarían un cambio de las lógicas hegemónicas de producción de conocimiento (desarrollo de tecnologías sociales) fueron introducidos de forma general y en una posición marginal frente a otros asuntos considerados fundamentales, no comprometiendo las jerarquías tradicionales de la política. 
La anterior afirmación no implica no considerar que la inclusión de estos temas entre los objetivos nacionales de la PCTI es un hecho de relevancia, al colocarlos en la agenda pública para su consideración a nivel discursivo y ejecutivo; esto era inexistente en las planificaciones previas a 2004. Lo mismo puede decirse de la apertura a la participación, aunque incipiente y moderada, de nuevos actores sociales en los procesos de planificación que habilitaron esta inserción temática.

En la experiencia de Uruguay estos temas también se introducen como prioridades generales y amplias, sin delimitar metas específicas, lo cual dificulta la evaluación de los resultados de la aplicación de las políticas en relación a las metas planteadas.

En el PENCTI se estableció como quinto principio rector el desarrollo de "innovaciones" sociales y se introdujo como tercer objetivo general del plan el desarrollo de capacidades para la apropiación social del conocimiento y la innovación inclusiva.

En cuanto a la concepción teórica que está en la base de los documentos, el caso brasilero puede enmarcarse en la perspectiva de la "economía del conocimiento", donde el cambio tecnológico y la innovación serían los motores que hacen posible la expansión y el crecimiento económico del país. A través de los documentos oficiales se promueve una visión de fortalecimiento de las capacidades de CTI con el fin de convertirse en potencia científica, tecnológica e innovadora. También desde una concepción de sistema nacional de innovación (SNI) se le otorga un peso importante a la innovación empresarial. Se promueve, de esta forma, desde la planificación en CTI una articulación estrecha con la política industrial.

En ambos países se explicita la necesidad de impulsar un desarrollo intensivo en conocimiento frente a una estructura productiva con gran peso de la producción de commodities.

En el caso uruguayo, por su parte, se encuentra además, en lo que respecta específicamente a inclusión social, una fuerte influencia del enfoque de sistemas de innovación socialmente orientados. Mientras que, en la ENCTI brasilera, la noción de inclusión social vinculada a CTI se presenta equiparada a las visiones de "transferencia", "diseminación" y "acceso" al conocimiento.

En este aspecto, en los planes de Uruguay prima una lógica similar de desarrollo tecnológico para la resolución de problemas de inclusión social, donde se propone generar y aplicar conocimientos desde los ámbitos académicos y técnicos para la resolución de estos problemas, contemplando a las poblaciones afectadas por los mismos solo en el momento de identificación de la demanda. No se plantea, por tanto, la posibilidad de participación de 
estos actores, que serán beneficiados por las soluciones tecnológicas propuestas, en el proceso de elaboración.

En cuanto a los instrumentos de política que responden a los objetivos de сті para la inclusión y el desarrollo social, se encuentran diferencias considerables entre ambos países.

En el caso brasilero está presente una amplia diversidad de programas e instrumentos con esta orientación dentro de la Secretaría de Ciencia, Tecnología e Innovación para la Inclusión Social (secis) del Ministerio de СтI, desde su origen en 2004. Mientras tanto, en Uruguay es posible encontrar, en el período 2008-2016, tres instrumentos, relacionados con la ejecución del Plan Estratégico a nivel nacional, por la agencia correspondiente del Estado (Agencia Nacional de Investigación e Innovación, ANII) que no presentan continuidad en todo el período.

La diversidad de programas presentes en el мСті brasilero puede estar relacionada con la existencia de una diversidad de actores con concepciones diferentes sobre exclusión/inclusión social presentes en la disputa de poder de la РСтr. Dicha diversidad genera una cierta imprecisión en los contenidos de los instrumentos, aludiendo a diversos temas y con distintas orientaciones.

En comparación con la diversidad de temas abordados por los instrumentos en el caso de Brasil dentro de la PCTDs, en Uruguay el abanico es mucho más restringido. Es decir, en la ANII uruguaya no se encuentra esa misma imprecisión en los instrumentos pero si un número muy escaso de instrumentos que se aplican con regularidad. La discusión llevada a cabo para la elaboración del PENCTI, así como la posterior implementación de los instrumentos de política, no dieron lugar ni se relacionaron a la generación de una red de actores trabajando en el tema, ni al desarrollo de otras experiencias dentro de organismos estatales o en articulación con la sociedad civil, a diferencia de lo ocurrido en Brasil.

En relación a los montos financieros invertidos en estos programas, se constata la escasa inversión realizada con esta orientación en el marco de la política general de сті en ambos países. Sin embargo, la diferencia en inversión brasilera con respecto a la uruguaya es considerable, promediando el $6 \%$ del presupuesto anual del MCTi en todo el período analizado, lo cual representa aproximadamente el doble de la cifra para Uruguay, que en todo el período no llega a superar el 3\% del presupuesto total de ANII.

Por tanto, en la experiencia brasilera la voluntad política, fuertemente ligada a la prioridad que representaban estos temas en el programa del PT, además de la mencionada vinculación con la sociedad civil, se hace más evidente que en el caso uruguayo en el desarrollo e implementación de estos instrumentos. 


\section{CONSIDERACIONES FINALES}

En el presente trabajo se abordó un análisis específico del actual desarrollo de un nuevo campo de acción de las políticas de ciencia, tecnología e innovación o, dicho de otra manera, de la nueva orientación de tales políticas, que introduce y da mayor énfasis a los temas de inclusión y desarrollo social, en su agenda política y, por derivación en planes y programas de сті.

Este análisis se particularizó en la situación de Brasil y Uruguay a partir de la asunción de los gobiernos progresistas de este siglo, mediante un estudio comparado. De esta manera, se analizó la introducción de los temas de inclusión y desarrollo social en la agenda de política pública y en los planes y/o estrategias nacionales de ciencia, tecnología e innovación (así como en documentos que dan origen y sustento a los mismos) en ambos países.

Se pudo constatar que el contexto político favorable para la incorporación del tema de сті para la inclusión y el desarrollo social, propiciado por la asunción de gobiernos progresistas y la incorporación en sus agendas de dichos temas como fundamentales, no fue suficiente para consolidar una política efectiva en la materia, más allá de la inclusión en el discurso, en los documentos oficiales y en los planes de ambos gobiernos.

Se encontraron similitudes y diferencias considerables entre ambos países. Como similitudes se encuentra, en primer lugar, el propio punto de partida: el contexto político favorable para la inserción de estos temas determinado por la asunción de gobiernos de perfil político progresista en ambos países (Partido de los Trabajadores y Frente Amplio, respectivamente), como condición de alguna manera necesaria. Por otra parte, en ambos países se da un acercamiento a procesos participativos y deliberativos en la elaboración de planes o estrategias nacionales de CтI, aunque con la diferencia de que en el caso brasilero este tipo de procesos se da de forma más amplia y con participación de organizaciones sociales además de los actores clásicos que discuten sobre estas políticas (técnicos del Estado y académicos, a veces representantes del medio productivo).

Como derivación de los elementos anteriores, en ambos países se encuentra una alta prioridad asignada a estos temas en los objetivos generales de los planes y estrategias nacionales efectivamente aprobados. Sin embargo, en relación al contenido de esos objetivos encontramos algunas diferencias: en Brasil se alude, en los documentos, a acciones y temas específicos, y en Uruguay la formulación del contenido del Plan es más general, dificultando la medición de resultados.

Por otro lado, las concepciones teóricas de base que orientan los planes en los aspectos específicos de inclusión social son diferentes. En el caso de 
Uruguay se constata un sustento teórico vinculado a la perspectiva de sistemas de innovación socialmente orientados. Mientras tanto, en el caso de Brasil, en lo que respecta al tema específico de inclusión social, no se encuentra sustentación conceptual vinculada a los enfoques teóricos desarrollados en la región.

En cuanto a los instrumentos y montos financieros invertidos en ellos, se constata una escasa inversión en ambos países pero la cifra relativa invertida en el marco de la PCTDs brasilera es de aproximadamente el doble en promedio que la inversión realizada en Uruguay.

Si bien no corresponde al período comprendido por este trabajo, es pertinente mencionar que en los últimos años, a partir de la asunción del presidente Temer en 2016, se produjeron cambios considerables en estos aspectos como la fusión de la SECIS con la SEPED y sus implicancias en cuanto a la priorización de los temas de popularización de la CTI e inclusión social (Natal y Alvim, 2018: 84).

En suma, para que la producción de conocimiento científico y tecnológico esté efectivamente al servicio de estrategias de desarrollo e inclusión social, no alcanza con la incorporación de nuevos actores al proceso decisorio de la PCTI (tal como se dio en la experiencia de Brasil), llevando a la inclusión del tema en la agenda y los planes. Es preciso ir más allá, promoviendo un cambio en la racionalidad de los actores dominantes para incrementar su compromiso con estos temas, un cambio más global en la orientación de la política de CTI.

Para que se produzca un cambio en la conceptualización, el enfoque y el marco de la сті fuertemente vinculado al desarrollo social incluyente es necesario un cambio en el paradigma de la política pública y la conexión explícita con estrategias de desarrollo (Sagasti, 2011). Por tanto, es necesaria una nueva agenda de política Сті fuertemente vinculada al desarrollo inclusivo (Bortagaray y Gras, 2013: 8).

\section{REFERENCIAS BIBLIOGRÁFICAS}

Albornoz, B., H. Thomas y J. Picabea (orgs.) (2015), Políticas tecnológicas y tecnologías politicas. Dinámicas de inclusión, desarrollo e innovación en América Latina, Bernal, UNQ / FLACso Ecuador.

Albornoz, M. (2007), "Los problemas de la ciencia y el poder", Revista Iberoamericana de Ciencia, Tecnología y Sociedad, 3, (8), pp. 47-65. 
Alzugaray, S., L. Mederos y J. Sutz (2013), "Investigación e innovación para la inclusión social: la trama de la teoría y de la política”, Isegoría. Revista de Filosofía moral y politica, 1, (48), pp. 25-50.

Arancibia, E. (2017), "Ciencia, tecnología e innovación para la inclusión social: un análisis de las experiencias de políticas públicas en América Latina”, tesis para obtener título de doctorado en Política Científica y tecnológica, San Pablo, unicamp.

Arbix, G. (2010), "Estrategias de Inovação para o desenvolvimento", Tempo Social, 22, (2), pp. 167-185.

Banco Mundial (2013), La movilidad económica y el crecimiento de la clase media en América Latina, Washington, Estudios del Banco Mundial sobre América Latina y el Caribe.

Bértola, L. et al. (coords.) (2005), "Ciencia, tecnología e innovación en Uruguay: diagnóstico, prospectiva y política”, Serie Documento de Trabajo de Rectorado, 26, Montevideo.

Bianchi, C. y M. Snoeck (2009), "Ciencia, tecnología e innovación en Uruguay: desafíos estratégicos, objetivos de política e instrumentos", Propuesta para el PENCTI 2010-2030, Montevideo, ANII.

Bortagaray, I. y N. Gras (2013), "Políticas de ciencia, tecnología e innovación para el desarrollo inclusivo: Tendencias cambiantes en América del Sur", en Crespi, G. y G. Dutrenit (eds.), Políticas de ciencia, tecnología e innovación para el desarrollo: La experiencia latinoamericana, México, Foro Consultivo Científico y Tecnológico-LALICS.

Bresser Pereira, L. (2007), "Estado y mercado en el nuevo desarrollismo", Nueva Sociedad, 210, pp.110-125.

Casas, R., J. M. Corona y R. Rivera (2014). "Políticas de ciencia, tecnología e innovación en América Latina: entre la competitividad y la inclusión social", en Kreimer, P. et al. (coords.), Perspectivas latinoamericanas en el estudio social de la ciencia, la tecnología y el conocimiento, México, Red Cyted / FCCyT / Siglo XXI, pp. 264-352.

cepal (2014), Panorama Social de América Latina 2014, Santiago de Chile, LC/G.2635-P.

Dagnino, R. (2007), "Empezando por la extensión universitaria", conferencia presentada en II Seminario Iberoamericano de Ciencia y Tecnología para el Hábitat Popular, Córdoba, Argentina.

(2008), Neutralidade da ciência e determinismo tecnológico, Campinas, Editora da UNICAMP.

Dias, R. (2012), Sesenta años de política científica y tecnológica en Brasil, Campinas, Editora da UNICAMP. 
Fonseca, R. (2009), Politica científica e tecnológica para o desenvolvimento social: uma análise do caso brasileiro, Campinas, DPCT/IG/UNICAMP.

Gabinete Ministerial de la Innovación (2007), Plan Estratégico Nacional de Ciencia, Tecnología e Innovación. Lineamientos fundamentales para la discusión, Montevideo, GMI.

- (2010), Plan Estratégico Nacional de Ciencia, Tecnología e Innovación, Montevideo, GMI.

Gaudichaud, F., J. Webber y M. Modonesi (2019), Los gobiernos progresistas latinoamericanos del siglo XXI. Ensayos de interpretación histórica, México, UNAM.

Herrera, A. (1973), La creación de tecnología como expresión cultural, México, Comercio Exterior.

Johnson, B. y B. Lundvall (1994), "Sistema nacional de innovación y aprendizaje institucional", Revista de Comercio Exterior, 44, (8), México, Bancomext.

Lanzaro, J. (2008), "La socialdemocracia criolla”, Nueva Sociedad, 217, Buenos Aires. Disponible en <https://nuso.org/articulo/la-socialdemocraciacriolla/>.

Lechini, G. (Comp.) (2008), La globalización y el Consenso de Washington: sus influencias sobre la democracia y el desarrollo en el sur, Buenos Aires, CLACSO.

Lundvall, B. (1992), National System of Innovation: Towards a Theory of Innovation and Interactive Learning, Londres, Pinter.

мсті (2007), Ciência, tecnologia e inovação para o desenvolvimento nacional: Plano de Ação 2007-2010, Brasilia, Ministério da Ciência e Tecnologia.

- (2010), Livro Azul da 4ta. Conferencia Nacional de Ciencia, Tecnologia e Inovacao, Brasilia, Ministério da Ciência e Tecnologia e Inovação.

- (2012) Estratégia Nacional de Ciência, Tecnologia e Inovação 2012-2015, Brasilia, Ministério da Ciência, Tecnologia e Inovaçáo.

Natal, C. y M. Alvim (2018), "A divulgação científica e a inclusão social", Revista do Edicc, 5, (8), pp. 76-86.

OCDE (1997), Science, Technology and Industry, París, Scoreboard of Indicators. Pereira, G y P. Escada (2012), "Participação da sociedad civil na IV Conferência Nacional de Ciência, Tecnologia e Inovação”, Liinc em Revista, 8, (3), pp. 52-67.

Rubianes, E. (2009), "Hacia una política de Estado en investigación e innovación”, en Elissalde, R. (comp.), Gozos y sombras del gobierno progresista. Aportes al balance, Montevideo, Ed. Dedos.

Sader, E. (2009), El nuevo topo. Los caminos de la izquierda latinoamericana, Buenos Aires, CLACso-Siglo XXI. 
Serafim, M. y R. Dagnino (2011), "A política científica e tecnológica e as demandas da inclusão social no Governo Lula (2003-2006)", Organizaçóes \& Sociedade, 18, (58).

Sutz, J. (2008), "Relaciones Universidad-empresa en América Latina", en Sebastián, J. (ed.), La evolución del desarrollo científico en América Latina, Madrid, Ediciones Fundación Carolina.

— (2010), "Ciencia, tecnología, innovación e inclusión social: una agenda urgente para universidades y políticas", Psicología, Conocimiento y Sociedad. Revista de la Facultad de Psicología, 1, (1), Montevideo, UdELAR.

Thomas, H., G. Bortz y S. Garrido, (2015), Enfoques y estrategias de desarrollo tecnológico, innovación y politicas públicas para el desarrollo inclusivo, Buenos Aires, IESCT-UNQ, CONICET.

Velho, L. (2011), La ciencia y los paradigmas de la politica científica, tecnológica $y$ de innovación, Campinas, UNICAMP.

Articulo recibido el 22 de octubre de 2019. Aprobado para su publicación el 30 de abril de 2020. 